Delft University of Technology

\title{
Vacuum assisted liquified metal (VALM) TSV filling method with superconductive material
}

Alfaro, J.A.; Sberna, P.M.; Silvestri, C.; Mastrangeli, M.; Ishihara, R.; Sarro, P.M.

DOI

10.1109/MEMSYS.2018.8346611

Publication date

2018

Document Version

Final published version

Published in

31st IEEE International Conference on Micro Electro Mechanical Systems (MEMS 2018)

\section{Citation (APA)}

Alfaro, J. A., Sberna, P. M., Silvestri, C., Mastrangeli, M., Ishihara, R., \& Sarro, P. M. (2018). Vacuum assisted liquified metal (VALM) TSV filling method with superconductive material. In 31st IEEE International Conference on Micro Electro Mechanical Systems (MEMS 2018): Belfast, Northern Ireland (Vol. 2018January, pp. 547-550). IEEE . https://doi.org/10.1109/MEMSYS.2018.8346611

\section{Important note}

To cite this publication, please use the final published version (if applicable).

Please check the document version above.

\section{Copyright}

Other than for strictly personal use, it is not permitted to download, forward or distribute the text or part of it, without the consent of the author(s) and/or copyright holder(s), unless the work is under an open content license such as Creative Commons.

Takedown policy

Please contact us and provide details if you believe this document breaches copyrights.

We will remove access to the work immediately and investigate your claim. 
Green Open Access added to TU Delft Institutional Repository 'You share, we take care!' - Taverne project

\section{https://www.openaccess.nl/en/you-share-we-take-care}

Otherwise as indicated in the copyright section: the publisher is the copyright holder of this work and the author uses the Dutch legislation to make this work public. 


\title{
VACUUM ASSISTED LIQUIFIED METAL (VALM) TSV FILLING METHOD WITH SUPERCONDUCTIVE MATERIAL
}

\author{
J.A. Alfaro ${ }^{1,4}$, P.M. Sberna ${ }^{2}$, C. Silvestri ${ }^{2}$, M. Mastrangeli $^{1}$, R. Ishihara ${ }^{3}$ and P.M. Sarro ${ }^{1}$ \\ ${ }^{1}$ Electronic Components, Technology and Materials, Delft University of Technology, Delft, NL \\ ${ }^{2}$ Terahertz Sensing, Delft University of Technology, Delft, NL \\ ${ }^{3}$ Quantum Information, Delft University of Technology, Delft, NL \\ ${ }^{4}$ Instituto Tecnológico de Costa Rica, Cartago, CR
}

\begin{abstract}
A novel, simple, low-cost method for the void-free filling of high aspect ratio (HAR) through-silicon-vias (TSVs) is presented. For the first-time pure indium, a type-I superconductor metal, is used to fill HAR vias, 300 to $500 \mu \mathrm{m}$ in depth and 50 to $100 \mu \mathrm{m}$ in diameter. The low electrical resistivity achieved without sintering, its reproducibility and straightforward processing steps, and the short time required to fill large arrays of vias at wafer scale - all make this method one of the simplest and quickest options for filling HAR TSVs for MEMS 3D integration. Moreover, the low melting point $\left(\sim 150{ }^{\circ} \mathrm{C}\right)$, malleability and superconductivity at $3.41 \mathrm{~K}$ make indium an interesting option in 3D interconnects for connecting quantum devices operating below $4 \mathrm{~K}$.
\end{abstract}

\section{INTRODUCTION}

System downscaling, 3D integration and increasing functionalities are the main challenges that both integrated circuits (ICs) and MEMS technology have been dealing with in the past decade. Advanced packaging schemes, low-cost materials and reliable interconnect technologies are therefore required. The scope of these requirements is moreover being currently extended to the quantum realm. Particularly, microwave resonant structures that store and manipulate quantum information units called qubits are used as base for quantum computers. Contrary to classic bits, qubits can assume during the computation any superposition of 0 and 1 states, and can interfere or be entangled according to quantum mechanics, thus enhancing the computational power for specific stochastic algorithms [1]. Physically, the qubits can be realized by any twolevel quantum system, such as e.g. excited states of an atom, nuclear and electron spins, positions of a crystalline defect, states of a quantum dot, or energy levels of a superconducting circuit [2]. To take full advantage of quantum computing, at least $10^{4}$ qubits have to be integrated and interconnected on the same mm-sized platform [3], avoiding any thermal disturbance of their coherent states and operations. Compact superconductive interconnections are ideally suited to help achieve this goal, as they would produce negligible thermal noise. 3D device integration through superconductive vias can afford just that.

Through-silicon vias (TSVs) technology is at the core of the heterogeneous integration of ICs with MEMS and NEMS (Fig. 1). Although the TSV technology using copper or aluminum as vias filling material is quite established, it still presents reliability issues such as mismatch among the coefficient of thermal expansion of the materials involved. TSV filling by electroplating and electroless plating is typically time-consuming and is hardly void- free, especially for high aspect ratio (HAR) vias [4]. Silver inkjet printing [5] and magnetic assembly of $\mathrm{Ni}$ rods $[6,7]$ have been recently reported to improve vias filling. However, these filling methods show limitations in substrate choice, via depth, processing time or temperature budget. In addition, the TSVs should achieve superconductivity to be suitable for the operations of quantum devices at cryogenic temperatures [8].

Here we propose a new method based on the combination of a novel filling material for vias, Indium, and a straightforward via filling technique named Vacuum Assisted Liquified Metal (VALM). The method allows to keep the temperature of the via filling process below 160 ${ }^{\circ} \mathrm{C}$, and achieves $\sim 90 \%$ yield and low resistivity interconnects without the need of additional thermal treatments. The proposed method thus enables the effective use of In as TSVs filling material, which in turn allows 3D MEMS integration also for superconductive devices. Indium achieves superconductivity at higher temperatures in comparison with Aluminum [9]. This is important in quantum engineering to ease the realization of a prototype of a quantum computer.

\section{TSV FABRICATION}

The TSV fabrication starts with either $300 \mu \mathrm{m}$ or 500 $\mu \mathrm{m}$-thick 4 inches Si wafers. A $6 \mu \mathrm{m}$ silicon dioxide layer is deposited on both wafer sides by a combination of thermal wet oxidation $\left(1100{ }^{\circ} \mathrm{C}\right)$ and plasma enhanced chemical vapor deposition $\left(400{ }^{\circ} \mathrm{C}\right)$. The silicon dioxide layer on the wafer frontside is used as hard mask during vias fabrication by deep reactive ion etching, DRIE. The $\mathrm{SiO}_{2}$ layer is patterned with circular structures, 50 to 100 $\mu \mathrm{m}$ in diameter, by reactive plasma etching with landing on the Si substrate.

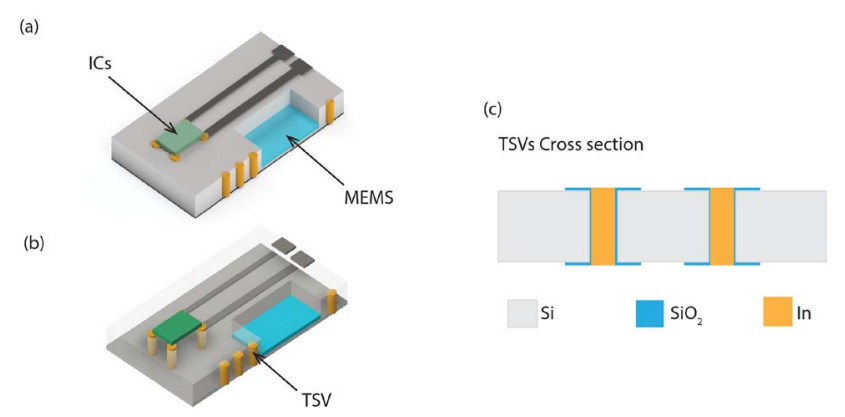

Figure 1. Sketches illustrating the heterogeneous integration concept including ICs and MEMS (a), TSVS integration (b) and schematic cross-section of the TSVS.

The TSVs are formed by DRIE of the silicon, landing on the silicon oxide at the wafer backside, and subsequent removal of the silicon dioxide left on both wafer sides by 
immersion in a $40 \%$ HF solution for 20 minutes. To provide the vias with the electrical insulation necessary to avoid mutual cross-talk and fading of RF signals, a 2.8 $\mu \mathrm{m}$-thick silicon dioxide layer is thermally grown on the surfaces of the vias, insulating the conductive core. Crosssection images of the achieved HAR TSVs for both 300 and $500 \mu \mathrm{m}$ thick wafers are reported in Fig. 2, showing the smooth sidewalls and straight profile of the vias.
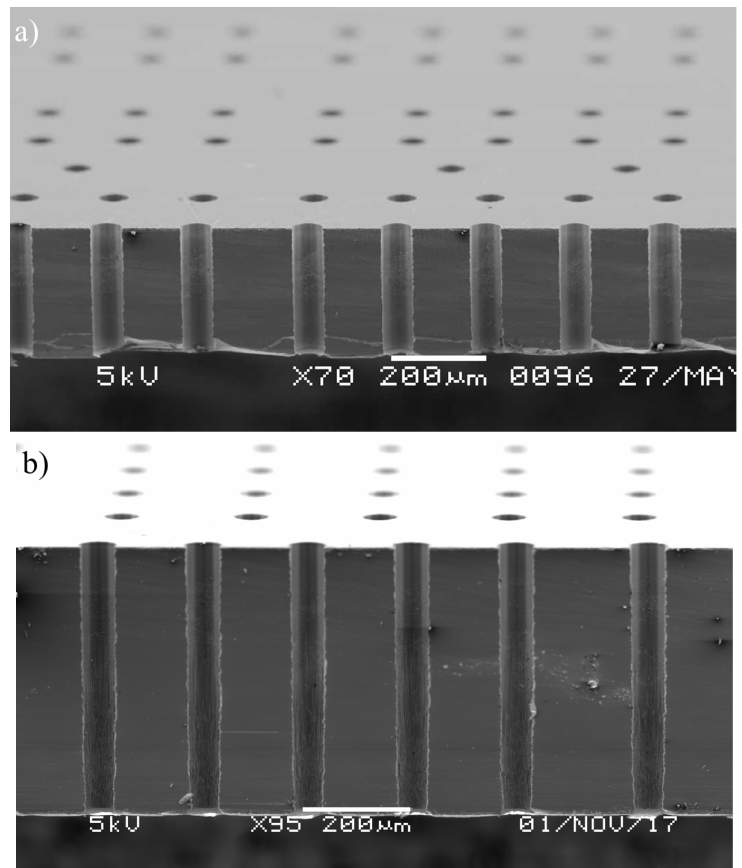

Figure 2: SEM images of TSVs cross-section after DRIE for a) $300 \mu \mathrm{m}$ deep and b) $500 \mu \mathrm{m}$ deep vias.

\section{VALM PROCESS}

The superconductive material used to fill the HAR TSVs is provided in the form of pure In beads $(6.35 \mathrm{~mm}$ diameter, trace metal basis higher than $99.99 \%$, Kurt J. Lesker Co. Ltd) with a melting temperature of $\sim 156^{\circ} \mathrm{C}$.

The VALM method designed to fill the TSVs is based on the combination of vacuum and heat supply using a custom-made tool (Fig. 3). The vacuum is generated in the tool by the Venturi effect and reaches a maximum value of -0.85 bar with an induced air flow of $28 \mathrm{nl} / \mathrm{min}$. The vacuum tool is placed on the hotplate which provides the heat to fully melt the In pellets and lower as much as possible its viscosity in the fluid state. The temperature shouldn't raise higher than $160{ }^{\circ} \mathrm{C}$, although it lowers the viscosity, to avoid In oxidation. Then the wafer is placed on the chuck of the vacuum tool. After waiting for temperature stabilization, the In beads are deposited on top of the wafer (Fig. 4a), whereby the In melts and is spread across the wafer surface. Subsequently the vacuum is activated. As a consequence, a pressure difference between the opposite sides/openings of the vias causes the molten In to flow inside the vias in a laminar flux regime with a specific velocity pattern, which is higher at the center of the via and lower towards its contour. According to the Stokes flow law, such pressure difference is a function of the center liquid velocity, liquid density, via dimeter and via length [10].
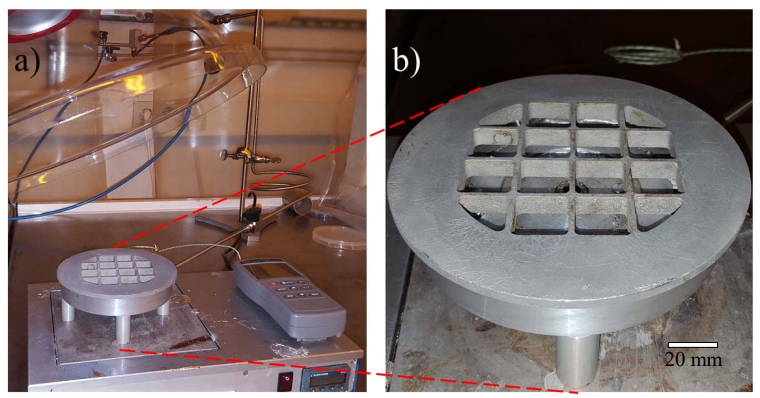

Figure 3: The custom-made setup for the VALM process (a), with close-up of the custom-made vacuum tool (b).

A blade, covered with a thin Kapton film, is then used to repeatedly scan the top wafer surface (Fig. 4b) to spread around the molten In into the vias until they are filled and a thin layer (few tens of $\square$ m-thick) is left on the surface (Fig. 4c). After blade coating, the vacuum is stopped, the wafer is covered with Kapton film for protection and turned upside down, so that the same procedure can be performed on the back side of the wafer (Fig. 4d). After complete filling of the vias, the In layer is patterned on both wafer sides using a conventional photolithographic process. A thick layer of photoresist is required to conformally coat the metal surface which presents topographical irregularities, caused by the manual blade coating and micrometric In oxide clumps which form upon exposure to air of the In surface. A $\sim 35 \mu \mathrm{m}$-thick photoresist layer (AZ9260, Microchemical $\mathrm{GmbH}$ ) was obtained by sequentially spray coating 32 layers of diluted photoresist (2 $\mu \mathrm{L}$ per layer). Finally, after resist exposure and development, the exposed In is etched in a solution of hydrochloric acid, hydrogen peroxide and water in a ratio of $8: 1: 1$ for around 7 minutes. (Fig. 4e).

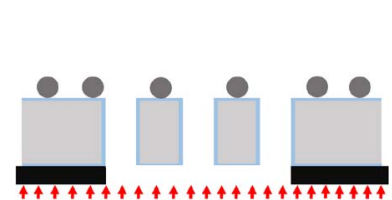

a)

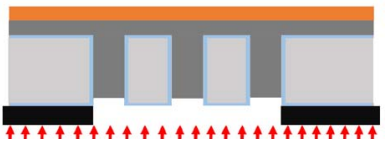

c)

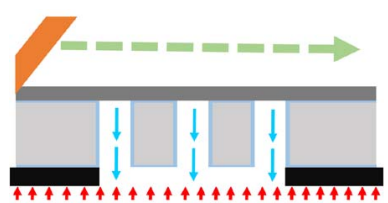

b)

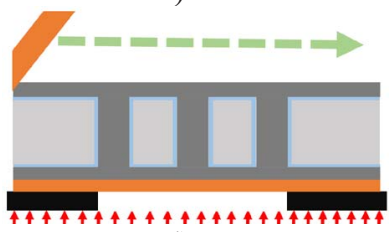

d)

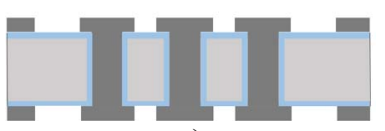

e)

Figure 4: Key process steps for the fabrication of In-filled HAR TSVs: a) Application of In beads over the DRIE etched wafer placed over the heated vacuum tool. b) Blade coating of melted In with activated vacuum. c) Vias filling with In, and coverage of top wafer side with Kapton film. d) In blade coating on bottom wafer side. e) Patterning of top and bottom In layers after complete vias filling. 


\section{VIAS CHARACTERIZATION}

To characterize the fabricated In-filled vias, electrical measurements were performed on the set of test structures shown in figure 5. A path of $200 \mu \mathrm{m}$ width by $200 \mu \mathrm{m}$ length was used for single via measurement. The same length was used for measurements with two vias in parallel and two vias in series with a separation between the vias of $180 \mu \mathrm{m}$. The width of the path was $445 \mu \mathrm{m}$ for the parallel configuration and $945 \mu \mathrm{m}$ for the TSVs in series.

The electrical resistivity of the achieved HAR TSVs In-filled through the VALM method was measured using an Agilent 4156C Semiconductor Parameter Analyzer and a Cascade probe station in a two-probe configuration. In particular, the measurements were performed by providing a stepwise voltage, from $-15 \mathrm{mV}$ to $+15 \mathrm{mV}$, at three different vias configurations: single via, two vias in parallel, and two vias in series (Fig. 6).

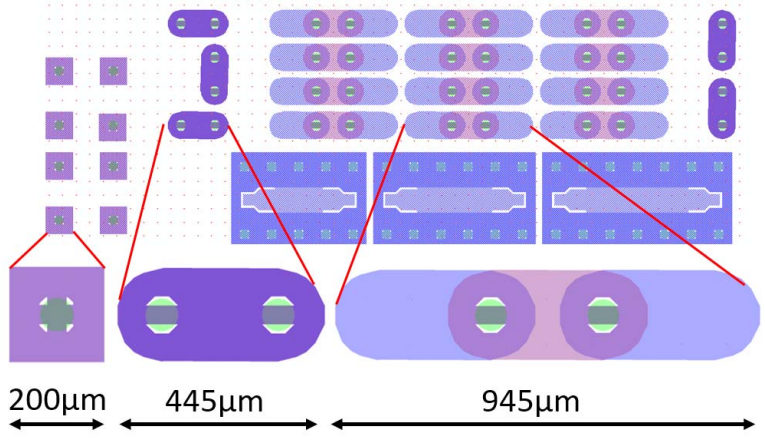

a)

b)

c)

Figure 5: Layout of the test structures for the electrical characterization of the indium vias: a) one single via; b) two vias in parallel; c) two vias in series
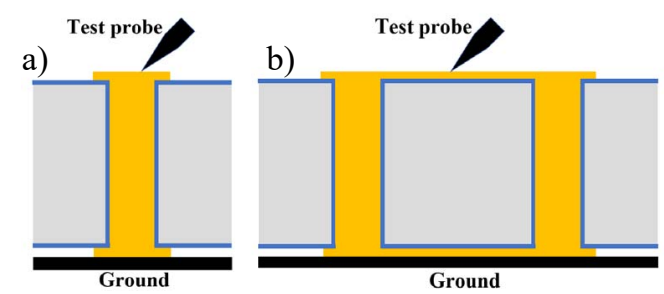

c)

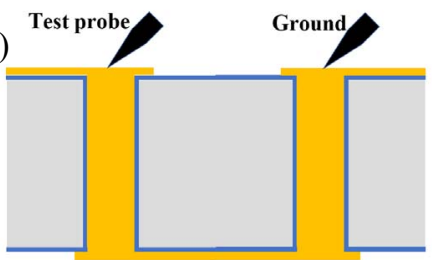

Figure 6: Configuration for the electrical characterization of the In-filled vias: a) single via; b) two vias in parallel; c) two vias in series.

\section{RESULTS AND DISCUSSION}

The VALM process allows to successfully fill HAR TSVs for both the $300 \mu \mathrm{m}$ and $500 \mu \mathrm{m}$ wafer thicknesses tested, as shown by the cross-section images reported in Figure 7. The voids-free filling of TSV has been obtained at wafer level in just 10 minutes, irrespective of the depth of the vias. In comparison, conventional via-filling methods such as electroplating and electro-less plating require around 20 hours and 2 hours, respectively [11]. The re- sults achieved show the effectiveness of the proposed VALM method for filling HAR TSVs, and open up the possibility of applying the method to other low melting point metals such as gallium, tin, eutectic alloys for soldering, and micro/nano suspension pastes.

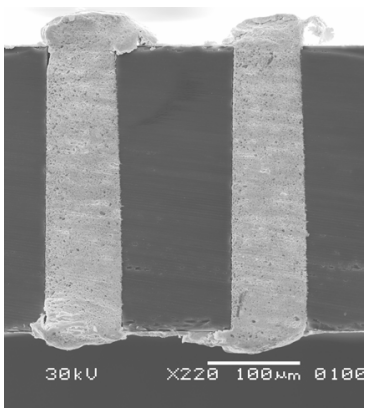

a)

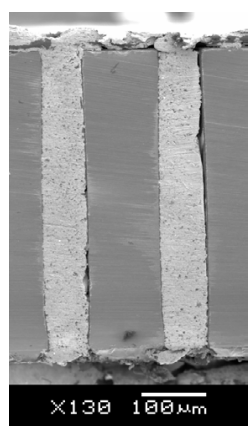

b)
Figure 7: SEM images of TSVs cross section after filling with indium a) $300 \mu \mathrm{m}$ deep vias b) $500 \mu \mathrm{m}$ deep vias. The voids and delamination in (b) were caused by the sawing used to obtain the cross-sections of the samples.
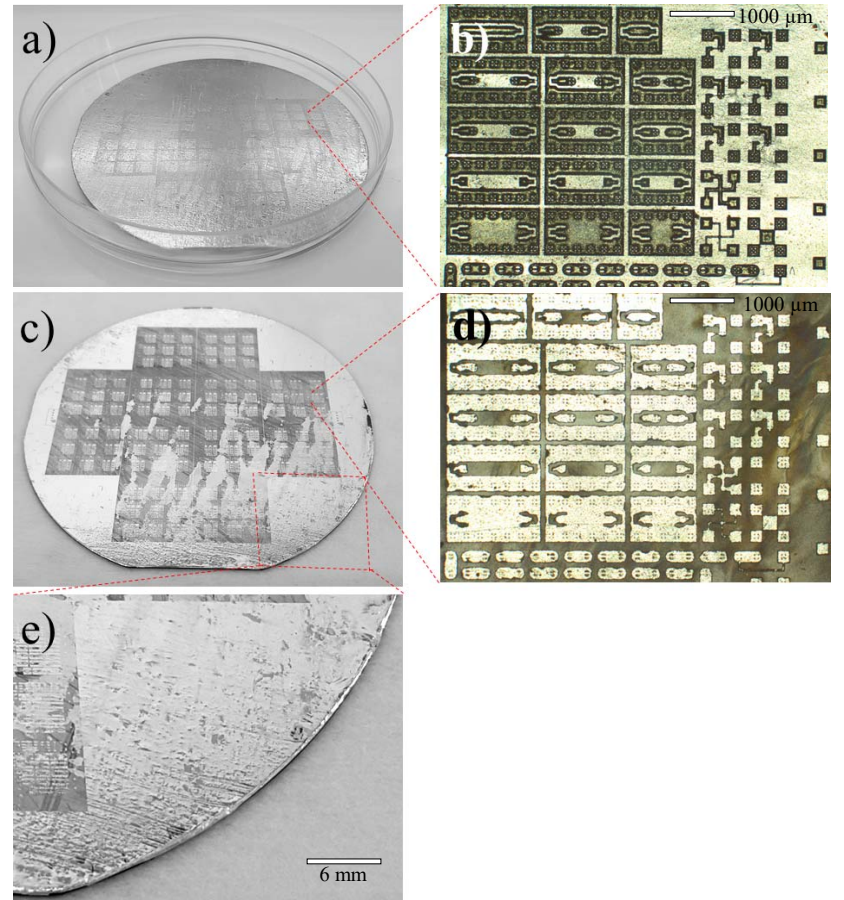

Figure 8: Patterning of indium after blade coating. Top view (a) and close-up (b) of the In-coated 4-inch wafer after photoresist patterning. Top view (c) and close-up (d) of patterned In test structures. e) Close-up of the surface roughness after In deposition.

Currently, the major challenge facing the fabrication process regards the etching of the In layers after via filling to define the interconnection lines and bondpads (Fig. 8). The challenge arises from the residual non-uniformity of the In layers after blade coating (Fig. 8c-e). It is crucial to achieve a good thickness uniformity after In spreading across the whole wafer surface to render the etching process more controllable. An ideal set-up would feature spin coating of molten In; a blade that could be actively heated, precisely aligned to the wafer surface and mechanical- 
ly moved; and an oxygen-free or forming gas environment to avoid In oxidation, such as a glove box.

The measurements for 75 um diameter vias in the 300 um-thick wafer are reported in Fig. 9. A resistance of 0.16 $\Omega$ was measured for the parallel configuration, and $3.60 \Omega$ for two vias connected in series (Fig 6). All the measured configurations show an ohmic behavior of the In-filled vias over the investigated voltage range. The obtained resistivity for a single via is as low as $593.6 \mu \Omega \bullet \mathrm{cm}$, which is in line with prior works $[5,6]$.

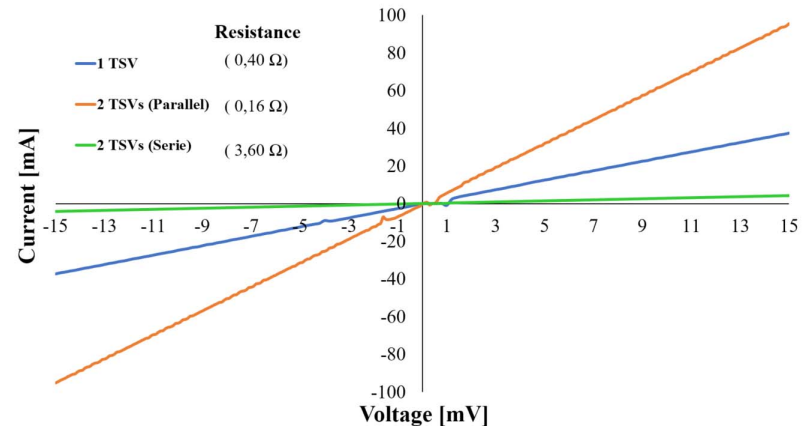

Figure 9: I-V curve of one TSV, two TSVS measured in parallel and two TSVs measured in series. An ohmic behavior is observed for the investigated voltage range.

\section{CONCLUSIONS}

We presented an effective and versatile method for filling high aspect ratio through silicon vias at wafer level with In, i.e. a low melting point and cryogenically superconductive material. HAR TSVs fabricated on both 300 and $500 \mu \mathrm{m}$-thick Si wafers have been used as test structures. The novel filling method, named Vacuum Assisted Liquified Metal (VALM), has been applied to fill voidfree TSVs in only 10 minutes irrespectively of vias depth. The TSVs filling yield is $\sim 90 \%$ for all diameter vias tested. Moreover, filling of high density TSVs is not an issue for this method, though via surface density is inherently restricted by the DRIE process. Indium patterning is challenged by the current non-uniformity of the blade coated In layer at the wafer surface. A dedicated tool designed to guarantee a uniform thickness of the molten layer is currently under construction. Two-terminal In-filled TSVbased structures were fabricated and electrically characterized. A single via resistance of $0.40 \Omega$ is measured for $300 \mu \mathrm{m}$ deep TSV and $75 \mu \mathrm{m}$ in diameter.

The simple and inexpensive method proposed to realize void-free, In-filled HAR vias is especially attractive for compact superconductive interconnects supporting quantum computing devices operating below $4 \mathrm{~K}$.

\section{ACKNOWLEDGEMENTS}

The authors gratefully acknowledge the technical support and advice of the staff at the TU Delft Else Kooi Laboratory. Special thanks to Loek Steenweg and Alex van der Bogaard for their help in the design and fabrication of the vacuum tool. Juan Andrés Alfaro-Barrantes is partially financed by Instituto Tecnológico de Costa Rica.

\section{REFERENCES}

[1] W. Pfaff, T.H. Taminiau, L. Robledo, H. Bernien, M.L. Markham, D. J. Twitchen, R. Hanson, "Demonstration of entanglement-by-measurement of solid state qubits," Nat. Phys., vol. 9, no. 1, pp. 29-33, 2012.

[3] J. M. Gambetta, J. M. Chow, and M. Steffen, "Building logical qubits in a superconducting quantum computing system," npj Quantum Inf., no. May 2016, 2015.

[4] A. Horibe, K. Sueoka, T. Aoki, K. Toriyama, K. Okamoto, S. Kohara, H. Mori, Y. Orii, "Through silicon via process for effective multi-wafer integration," in Proceedings - Electronic Components and Technology Conference, 2015, vol. 2015-July, pp. 18081812.

[5] T. Yang, T. Yang, Z. Guo, Y. Fu, Y. Cheng, Y. Song, $\mathrm{P}$. Wu, Synchrotron National, "A low temperature inkjet printing and filling process for low resistive silver TSV fabrication in a SU-8 substrate" Department of Electronics Engineering, National Chiao Tung University, Hsinchu, Taiwan Program of Science and Technology of Accelerator," 2017, pp. 749752.

[6] M. J. Laakso, M. Asiatici, A. C. Fischer, G. Stemme, and F. Niklaus, "Wide temperature range through silicon vias made of Invar and spin-on glass for interposers and MEMS," in Proceedings of the IEEE International Conference on Micro Electro Mechanical Systems (MEMS), 2016, vol. 2016-February, pp. 585-588.

[7] M. J. Laakso, J. Liljeholm, A. C. Fischer, G. Stemme, T. Ebefors, and F. Niklaus, "Maskless manufacturing of through glass vias (TGVS) and their test structures," in Proceedings of the IEEE International Conference on Micro Electro Mechanical Systems (MEMS), 2017, pp. 753-756.

[8] J. I. Colless and D. J. Reilly, "Modular cryogenic interconnects for multi-qubit devices," Rev. Sci. Instrum., vol. 85, no. 11, 2014.

[9] J. F. Cochran and D. E. Mapother, "Superconducting Transition in Aluminum," Phys. Rev., vol. 111, no. 1, pp. 132-142, 1958.

[10] Z. Dagan, S. Weinbaum, and R. Pfeffer, "An infiniteseries solution for the creeping motion through an orifice of finite length," J. Fluid Mech., vol. 115, no. 1, p. 505, 1982.

[11] F. Santagata, G. Fiorentino, M. Nie, C. Farriciello, R. Poelma, G. Q. Zhang, P.M. Sarro, M. Nie, "Fully back-end TSV process by $\mathrm{Cu}$ electro-less plating for 3D smart sensor systems," in Proceedings of IEEE Sensors, 2012.

\section{CONTACT}

*J.A. Alfaro, tel: +31-06-53473356; a.alfarobarrantes@tudelft.nl 